\title{
Confocal Microscopy Visualizes Particle-Crack Interactions in Epoxy Composites with Optical Force Probe-Crosslinked Rubber Particles
}

\author{
Maria Stratigaki, ${ }^{a}$ Christoph Baumann, ${ }^{a, b}$ and Robert Göst|*a
}

\begin{abstract}
${ }^{a}$ DWI - Leibniz Institute for Interactive Materials, Forckenbeckstr. 50, 52056 Aachen, Germany
b Institute of Technical and Macromolecular Chemistry, RWTH Aachen University, Worringerweg 1, 52074 Aachen, Germany
\end{abstract}

*goestl@dwi.rwth-aachen.de 


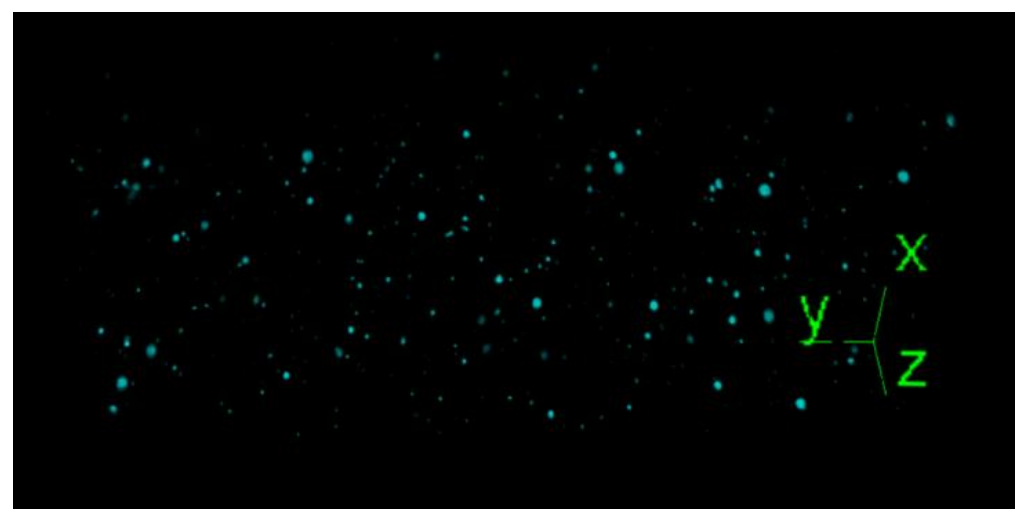

Figure S1. CLSM snapshot based on a 3D reconstruction (Movie S1) showing the dispersion state of the fluorescent inclusions in the composite films. The animated $3 \mathrm{D}$ reconstruction under $360^{\circ}$ rotation was prepared with the 3D viewer plugin of Fiji (volume display with resampling factor of 2).

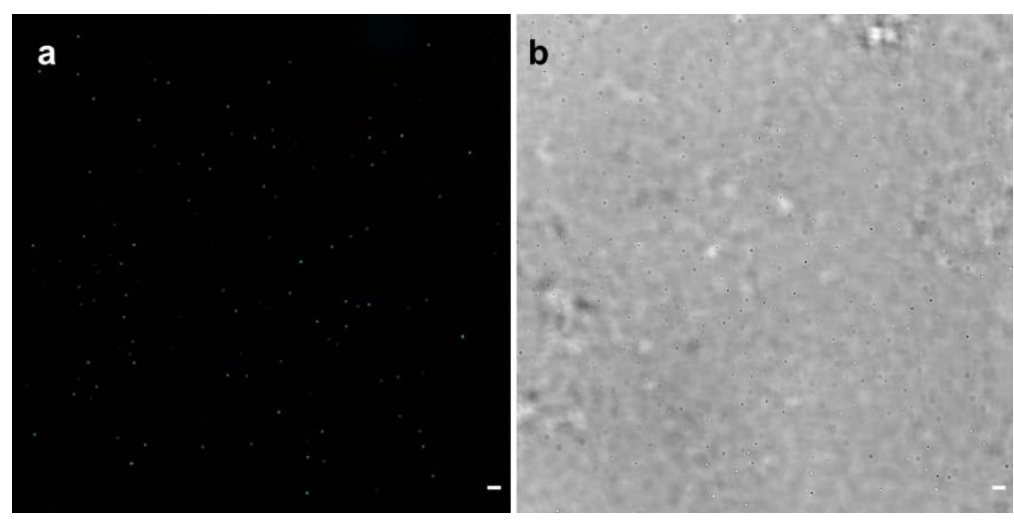

Figure S2. CLSM images showing particles produced with the addition of surfactant during emulsion polymerization with narrow size distribution after dispersion in epoxy matrix. Scale bar: $5 \mu \mathrm{m}$. 

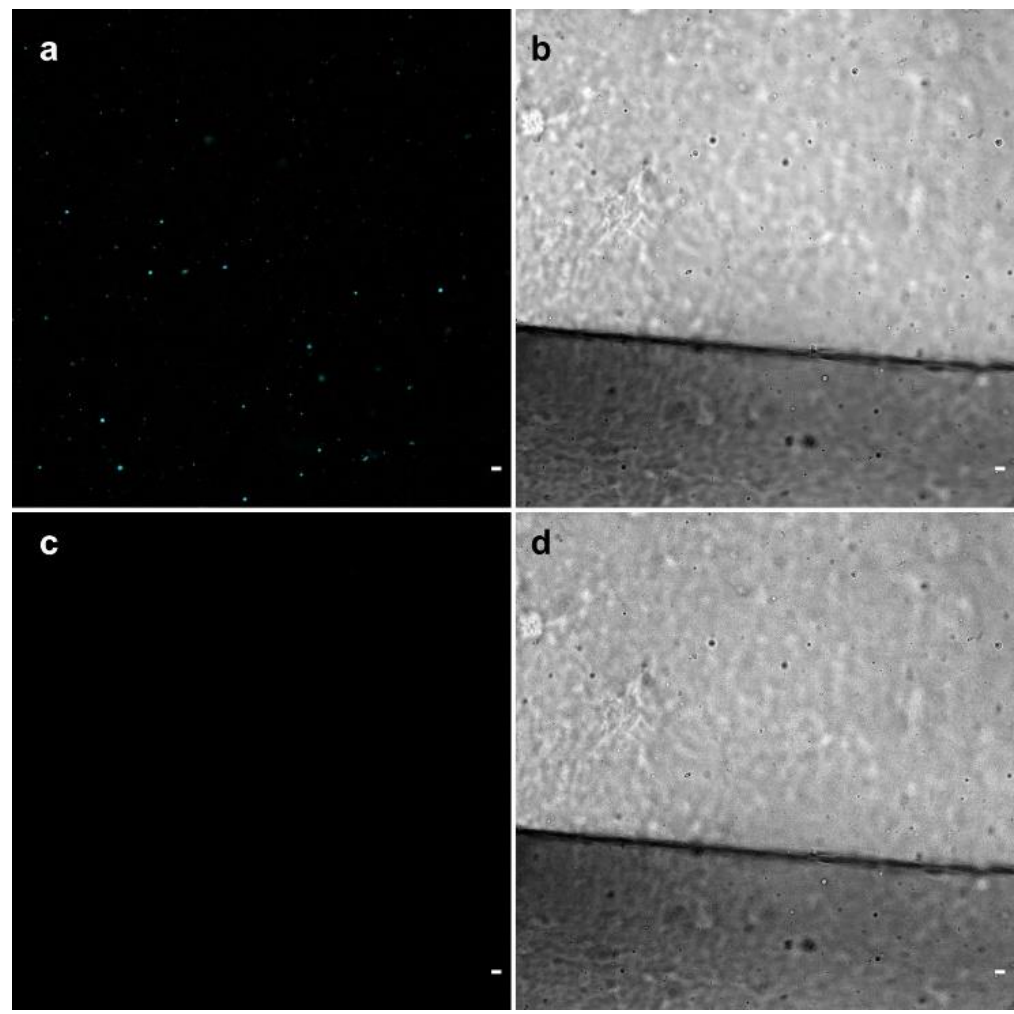

Figure S3. CLSM images showing fluorescence $(a, c)$ and brightfield $(b, d)$ modes of dispersed particles in epoxy matrix in their pristine, non-activated state exhibiting only cyan fluorescence (a) and not any yellow fluorescence (c) since not one of them is in an activated state, and corresponding (identical) images in the brightfield mode showing the particles. Scale bar: $5 \mu \mathrm{m}$. 